\title{
GERENCIA ESTRATÉGICA: HERRAMIENTA PARA LA TOMA DE DECISIONES EN LAS ORGANIZACIONES
}

\section{Strategic management: tool for decision making in organizations}

Jorge González*; Flor Salazar**; Raúl Ortiz ${ }^{* * *}$ y Darwin Verdugo****

\section{RESUMEN}

La gerencia estratégica en las organizaciones reviste gran importancia, al ser quien define el direccionamiento del negocio en un futuro inmediato; en este sentido, las acciones que delinean tal direccionamiento, deben estar alineadas con el pensamiento de quienes se encuentran al frente de los negocios. El objetivo de este trabajo, consiste en analizar la gerencia estratégica en las organizaciones como herramienta para la toma de decisiones y responder a las exigencias del entorno competitivo. Se asume para efectos de la investigación desarrollada, una postura enmarcada en la metodología cualitativa, con enfoque documental, sentando las bases para una investigación de mayor alcance. Se revisan documentos especializados con el fin de realizar una disertación teórica inicial, tales como Thompson y Strickland (2004), David (2003); Chiavenato (2011), entre otros. Los resultados indican que la literatura especializada en el área es diversa y arrojan elementos esenciales para la construcción del pensamiento estratégico, este último caracterizado por el desarrollo de procesos críticos en la mente del estratega que coadyuvan a mejorar las capacidades de las organizaciones, en aras de formular y desplegar estrategias con mayor efectividad. El pensamiento estratégico no se concentra únicamente en la elaboración de planes, va más allá, permitiendo cambiar modelos mentales de quienes toman las decisiones en las organizaciones.

Palabras clave: Gerencia estratégica, pensamiento estratégico, organizaciones.

RECIBIDO: Marzo 2018

ACEPTADO: Octubre 2018

\footnotetext{
${ }^{*}$ Economista, Magíster en Auditoría Integral. Docente e investigador de la Universidad Católica de Cuenca, Ecuador. Correo electrónico: jgonzalezu@ucacue.edu.ec

** Ingeniera empresarial, Máster en Auditoría Integral, Máster en Docencia Superior. Docente e investigador de la Universidad Católica de Cuenca, Ecuador. Correo electrónico: fisalazarv@ ucacue.edu.ec

${ }^{* * * *}$ Ingeniero empresarial. Magister en Administración y dirección de empresas. Docente e investigador de la Universidad Católica de Cuenca, Ecuador. Correo electrónico:: raortizg@ ucacue.edu.ec

**** Abogado. Libre ejercicio profesional. Ecuador. Correo electrónico: papoverdugo@ hotmail.com
} 


\section{ABSTRACT}

The strategic management in the organizations is of great importance, as it is who defines the direction of the business in the immediate future; In this sense, the actions that outline such direction must be aligned with the thinking of those who are in charge of the business. The objective of this work is to analyze strategic management in organizations as a tool for decision making and respond to the demands of the competitive environment. It is assumed for the purposes of the research developed, a position framed in qualitative methodology, with a documentary approach, laying the foundations for a more far-reaching investigation. Specialized documents are reviewed in order to carry out an initial theoretical dissertation, such as Thompson and Strickland (2004), David (2003); Chiavenato (2011), among others. The results indicate that the specialized literature in the area is diverse and throw essential elements for the construction of strategic thinking, the latter characterized by the development of critical processes in the mind of the strategist that help to improve the capacities of organizations, in order to formulate and deploy strategies with greater effectiveness. Strategic thinking does not concentrate only on the elaboration of plans, it goes further, allowing to change mental models of decision-makers in organizations

Keywords: Strategic management, strategic thinking, organizations

\section{Introducción}

La globalización nos obliga a reconocer que el mercado es todo el planeta, que se requiere un diseñar planes de negocio que tomen en cuenta los recursos no importa donde estén, clientes de todos los continentes, proveedores de otras latitudes, todo para optimizar la acción empresarial. Por cuanto es importante considerar a la economía de mercado como el campo natural donde las empresas se desempeñan.

Ante estos desafíos, las organizaciones tienen la obligación de ser lo suficientemente flexibles para transformar toda la estructura de negocios, y así responder a cambios estratégicos y retos del mercado. En tal sentido, para alcanzar una posición de ventaja competitiva, se necesita examinar de manera crítica el entorno, con el objetivo de identificar oportunidades externas y crear capacidades internas; se ha pasado de ser organizaciones estáticas a insertarse en un mundo dinámico, con altas exigencias que demandan una visión sistémica e integral.

En virtud de ello, se hace indispensable que las organizaciones, deban estar 
continuamente escudriñando el entorno, y en particular, el sector en el cual la organización se encuentra envuelta, debido a que el mismo define posteriormente una dirección estratégica a través de una secuencia de diferentes decisiones.

En este sentido, el enfoque de la gerencia estratégica contribuirá en forma definitiva a valorar el entorno, la competencia, las regulaciones gubernamentales, los avances tecnológicos, como variables críticas para asegurar la sobre vivencia y porque no decirlo el éxito de las empresas.

La Gerencia Estratégica, es una herramienta para administrar y ordenar los cambios, donde se definen los objetivos de la organización y se establecen estrategias; así mismo, se reconoce la participación basada en el liderazgo y la toma de decisiones que correspondan alas demandas del ambiente inmediato y futuro.

Los cambios organizacionales, han evolucionado a medida que se generan modificaciones mentales y estructurales en el hombre, a fin de lograr ser sostenibles en el tiempo. La velocidad con que se generan los cambios a través de los años es cada vez más vertiginosa, generando en las empresas la necesidad de estar atentos a las tendencias del mercado, en las cuales los clientes cada día poseen más información debido a la facilidad de su acceso.

Así mismo, para que las organizaciones puedan alcanzar ventajas competitivas y ser sostenibles es necesario ser dinámicas dentro de su plan estratégico para lograr la capacidad de crear conocimiento, transferirlo entre sus integrantes (diferentes áreas funcionales que conforman la organización), a fin de que se desarrolle la aplicación del conocimiento.

Es importante destacar lo que señala Ohmae (2004), el pensador estratégico divide en sus partes constitutivas lo que considera problemas, tendencias, acontecimientos o situaciones que parecen venir integradas como un todo, y tras descubrir el significado de estos componentes, los vuelve a ensamblar para maximizar sus ventajas, lo cual le permite encontrar respuestas realistas a situaciones cambiantes.

Lo anterior, conlleva al desarrollo de la empresa en su entorno o medio ambiente, conociendo, desarrollando y aplicando la gerencia estratégica como un arma 
Jorge González; Flor Salazar; Raúl Ortiz y Darwin Verdugo

Telos Vol. 21, No. 1 (2019). 242-267

poderosa en el mundo competitivo de hoy, basadas en factores como: ¿qué productos y servicios ofrecer?, ¿qué demandas del mercado satisfacer?, ¿qué segmento de clientes atender?, ¿qué tecnología utilizar? (o desarrollar), ¿qué método de ventas y distribución utilizar?, y ¿qué área geográfica atacar?

En este sentido, el gerente debe asumirse como un agente de cambio y de transformación, que conduzca a las organizaciones por nuevos rumbos, nuevos procesos, nuevos objetivos, nuevas estrategias, nuevas tecnologías e incluso nuevos riesgos; un agente que con su dirección y orientación, modifique comportamientos y actitudes del capital humano, un agente cultural que, con su estilo de gerencia, reforme y difunda la cultura organizacional, utilizando técnicas de desarrollo gerencial dirigidas a incrementar la habilidad del liderazgo y resaltar los valores institucionales.

Para García (2010), el propósito del pensamiento estratégico es ayudar a las organizaciones, en la exploración de los desafíos futuros, tanto previsibles como imprevisibles, más que preparar a las mismas para un posible mañana único. En consecuencia, el pensamiento estratégico de la empresa es la coordinación de varias mentes creativas dentro de una perspectiva común que le permita a un negocio avanzar hacia el futuro de una manera satisfactoria para todos.

El Pensamiento Estratégico incorpora valores, misión, visión y estrategia que tienden a ser elementos intuitivos, basado en los sentimientos, más que analítico, basados en información. Llegar a un acuerdo sobre estos elementos entre los miembros del equipo administrativo es un prerrequisito esencial para la planeación efectiva. De aquí se infiere la importancia del pensamiento estratégico, y radica en que es el cimiento para la toma de decisiones estratégicas, sin este fundamento las decisiones y acciones subsecuentes quizá sean fragmentadas e inconsistentes para el buen desempeño a largo plazo de la empresa (Chiavenato y Sapiro, 2011).

Toda organización necesita tener un norte, directrices o políticas que laguíen, que faciliten a través del análisis, tanto interno como externo,saber cuáles son los objetivos que se espera obtener en el futuro y es allícuando surge la planeación 
estratégica, como parte de la gerencia estratégica para definir el alcance, lo que se quiereser y establecer, y cuál es la razón de ser de la empresa son algunos de esosaspectos importantes que van a permitir plantear las estrategias necesariaspara cumplir con lo que se espera.

En la organización se pueden analizar las estrategias con relación a cómo se van a implementar en el corto o largo plazo, con miras a lograr los objetivos propuestos en las políticas de la empresa. Pero también podrían revisarse desde el punto de vista de la sostenibilidad y del direccionamiento, a través del establecimiento de objetivos globales y específicos que lleven a obtener los mejores resultados con los recursos que se tienen.

Más aún, es probable que las estrategias se enfoquen en la competitividad y el entorno, y es ahí cuando muchos empresarios hacen ver lo valioso que es aprender y adaptarse a los cambios en forma más rápida que los competidores.

Esta investigación busca analizar como la gerencia estratégica como herramienta para la toma de decisiones en las organizaciones, or cuanto los gerentes deben delinear un direccionamiento en la organización que permita lograr ser más competitivos

Se asume para efectos de la investigación desarrollada, una postura enmarcada en una metodología cualitativa. Este estudio tiene como característica la revisión de textos, revistas científicas, tesis y otros documentos que son el resultado de otras investigaciones, reflexiones de teóricos, lo cual representa la base que sustenta la exploración; y, en tal sentido, el conocimiento se construye a partir de su lectura, análisis, reflexión e interpretación por parte del investigador.

A continuación se realizan algunas reflexiones teóricas sobre la gerencia estratégica, se describen algunos elementos que la constituyen, donde se desarrollan el diagnostico organizacional, la filosofía de gestión de las empresas, el direccionamiento estratégico y la evaluación estratégica, y, finalmente se realizan las reflexiones finales.

\section{La gerencia estratégica. Algunas reflexiones teóricas.}


Jorge González; Flor Salazar; Raúl Ortiz y Darwin Verdugo

Telos Vol. 21, No. 1 (2019). 242-267

La administración estratégica, también denominada dirección estratégica, direccionamiento estratégico, gerencia estratégica o planificación estratégica, exige el análisis de las organizaciones desde la perspectiva integral, así como la adopción de visiones ampliadas, adoptando y adaptando por parte de los tomadores de decisiones, modelos mentales que faciliten armonizar la visión del mundo y de sus participantes (Chiavenato y Sapiro, 2011).

$\mathrm{Su}$ abordaje desde una concepción tradicional, se ha ido perfeccionando y complementando con visiones modernas, acompañadas de avances que se han ido incorporando en la literatura especializada (Thompson y Strickland, 2001; David, 2003; Chiavenato, 2001; Hitt y col., 2004; entre otros), esto incluye entre otros aspectos, definiciones, elementos y etapas básicas que integran y amplían la visión de la administración o gestión en las organizaciones actuales.

En este sentido, la administración estratégica es definida por autores diversos, estando estos en sintonía con la forma de concebirla, pues en la mayoría de los casos existen coincidencias en los elementos o aspectos claves considerados en su definición. Citando un clásico en el área, David (2003), plantea que esta puede ser definida como el arte y la ciencia de formular, implantar y evaluar las decisiones a través de las funciones que permiten a una empresa lograr sus objetivos; delinea por un lado la misión de la organización, mientras que por el otro lado su dirección futura, los objetivos estratégicos a largo plazo, a la vez que define estrategias y políticas para lograrlos; todo ello apoyado en procesos de evaluación del entorno tanto externo como interno a la organización.

Para David (2003), la administración estratégica es un proceso que inicia con el establecimiento de metas organizacionales, define estrategias y políticas para lograr estas metas, y desarrolla planes detallados para asegurar la implantación de las estrategias y así obtener los fines buscados. Permite tomar decisiones sobre el tipo de esfuerzos de planeación que deben hacerse, cuándo y cómo debe realizarse, quién lo llevará a cabo, qué se hará con los resultados. 
Según el autor, la planeación estratégica es sistemática en el sentido de que es organizada y conducida con base en una realidad entendida. Sin embargo, para la mayoría de las organizaciones, representa una serie de planes y acciones producidas después de un período de tiempo específico. Responde a un proceso continuo, particularmente en lo que respecta a la formulación de estrategias (David, 2003), esto debido a los cambios continuados del contexto.

En sintonía David (2003), establece que la administración estratégica, se concentra en la formulación de estrategias de una empresa basada en la creación de un conjunto de ventajas competitivas, como parte de un sistema de planeamiento sistemático.

Precisando lo anterior, Thompson y Strckland (2001), puntualizan que la administración estratégica debe asumirse como un proceso y no como un acontecimiento aislado de la vida organizacional, a la vez que establece cinco tareas: 1) desarrollo de una visión estratégica de la organización, precisando hacia dónde se dirige, 2) determinación de objetivos que permitan convertir la visión estratégica en resultados específicos del desempeño que se deberá alcanzar, 3) creación de estrategias que apunten al logro de objetivos, 4) puesta en práctica de la estrategia de manera eficiente y efectiva y, 5) evaluación del desempeño e inicio de ajustes correctivos en la visión, la dirección, los objetivos, la estrategia o la puesta en práctica, vista desde la experiencia real, de las condiciones cambiantes, de nuevas ideas y de las nuevas oportunidades.

Por su parte Francés (2006) comparte las definiciones anteriores al definirla como un proceso sistemático que establece lineamientos estratégicos para una organización. El autor precisa que este proceso toma en cuenta la incertidumbre mediante la identificación de oportunidades y amenazas en el entorno, y trata de anticipar lo que otros actores pueden hacer, destacando que la estrategia ejecutada se alimenta también de la emergente, que surge de la actividad diaria, y que se incorpora a la estrategia formulada.

Autores como Betancourt (2006) establecen que debe ser vista como el "arte y/o 
Jorge González; Flor Salazar; Raúl Ortiz y Darwin Verdugo

Telos Vol. 21, No. 1 (2019). 242-267

ciencia de anticipar y generar el cambio, con el propósito de crear permanentemente estrategias que permitan garantizar el futuro del negocio".

Rivas y Velásquez (2009) plantean que la gerencia estratégica, se puede concebir como una estructura teórica para la reflexión acerca de las grandes opciones de la empresa, reflexión que se fundamenta en una nueva cultura organizacional y una nueva actitud de la dirección, donde ya no se trata de copiar las dificultades devenidas de un entorno turbulento sino ir a su encuentro, donde se huye de lo improvisado en busca de lo analítico y de lo formal.

En este contexto, cabe expresar que la gerencia estratégica encaja dentro del concepto de organización inteligente introducido en 1990 por Peter Senge (2009), pero ya acuñado por Seymour Papert en la década de los '60, la cual involucra la estrategia a todos los sectores de la organización rompiendo el paradigma de los niveles de la administración científica; es decir, que este involucramiento de las personas en la gestión estratégica, y la internalización de la visión en cada uno de los miembros de la organización, es lo que convierte a una organización en inteligente.

La gerencia estratégica ha ido evolucionando en forma secuencial, siendo posible identificar fases específicas (Saavedra, 2005): 1) la atención de la administración general o tradicional: enfoque centrado en el control operativo de las actividades de la empresa, utilizando como base el planeamiento financiero básico; 2) planeamiento de actividades de manera más completa, agregándose el análisis ambiental y un proceso presupuestario y de asignación de recursos 3) se incorpora un análisis más completo respecto de los mercados objetivos que una empresa ha definido, proponiéndose una evaluación competitiva, se agrega en esta etapa una evaluación, desde diferentes puntos de vista, de las opciones que se le presentan a una empresa para lograr sus objetivos. La fase final para el autor, se denomina Administración Estratégica, consistente en la formulación de estrategias basadas en la creación de un conjunto de ventajas competitivas, como parte de un sistema de planeamiento sistemático. 
Al respecto, plantea Saavedra (2005), que la aplicación de la metodología de la administración estratégica, luego de su fase introductoria en la administración de empresas, logra desarrollar procesos sistematizados que permite estudiar diferentes escenarios futuros, conjuntamente con el análisis de las capacidades internas de la organización.

Con la gerencia estratégica, se desarrolla un pensamiento estratégico que constituye la parte no analítica del trabajo del estratega, la esfera sofisticada y compleja del conocimiento que implica imaginación, discernimiento, intuición, iniciativa, fuerza mental e impulso para aprender (Chiavenato y Sapiro, 2011). Para los autores, el pensamiento estratégico, es la forma en la que los estrategas visualizan el mundo exterior, situaciones desafiantes e inciertas, definiendo técnicas para la definición y resolución de problemas.

En este contexto, producto de la evolución del concepto, la administración estratégica adquiere características propias, que se puede definir e identificar por la calidad de los escenarios y eventos que se enfrentan, siendo éstos más dinámicos productos de situaciones del entorno variable y muchas veces turbulento (Day Y Reibstein, 1997; citado por Saavedra, 2005); esto aunado a movimientos que ocurren entre empresas rivales en un determinado mercado (Saavedra, 2005).

Durante el proceso evolutivo de la gerencia estratégica, este proceso de gestión logró sistematizar procesos organizacionales, contribuyendo en la formulación de la estrategia, el estudio del análisis del ambiente externo que enfrenta una organización a través de las categorías denominadas, oportunidades-peligros, en cuanto a las capacidades internas, fortalezas debilidades, de manera que se pueda formular una estrategia realista, para la obtención de los objetivos principales que ha definido la empresa. (Mintzberg, 1990).

También logró concentrar la atención en el desarrollo de visiones de negocio a largo plazo, siendo el calificativo de estratégico el que le permitirá ir definiendo y redefiniendo prácticas de gestión ante escenarios futuros complejos y con alto dinamismo. Es la encargada de definir misiones, visiones y objetivos, políticas y 
Jorge González; Flor Salazar; Raúl Ortiz y Darwin Verdugo

Telos Vol. 21, No. 1 (2019). 242-267

estrategias como parte del sistema gerencial de una organización, apoyado sin lugar a dudas en la diagnosis del contexto en el que se encuentran inmersas las organizaciones y complementado con el análisis minucioso de elementos internos que forman parte del sistema de gestión de las organizaciones.

En consecuencia, la gerencia estratégica se diferencia de la administración tradicional por el simple hecho, de que la primera logra que toda la organización se compenetre inequívocamente de la dirección que debe tomar y mantener la empresa; esto a pesar de la relación existente entre los conceptos tradicionales de administración y los incorporados bajo la visión estratégica de concebir las organizaciones (Saavedra, 2005).

\section{Elementos de la Gerencia Estratégica}

Al propiciar el análisis de la gerencia estratégica, se hace necesaria su desagregación, en miras a descomponer su esencia y precisar cada uno de los elementos que la integran. $\mathrm{Al}$ asumir la gerencia estratégica como un proceso, esta desprende ciertos elementos, también denominados tareas (Thompson y Strckland, 2004), etapas del modelo integral de dirección estratégica (David, 2003), entre otras connotaciones que ha recibido a través del tiempo. Lo importante, es que los autores (Thompson y Strickland, 2004; David, 2003; Hitt y col., 2004; Chiavenato, 2011 y 2001) coinciden en ellos, independientemente del nombre que reciban.

Es de destacar que el modelo de Gerencia estratégica presentado, subyace de estudios procedentes de la escuela de la planificación, la cual aborda la estrategia como un proceso formal (Chiavenato y Sapiro, 2011), a la vez, la escuela del diseño que asume la estrategia como un proceso de adecuación y la escuela del posicionamiento cuyo norte es la estrategia acogida desde un proceso analítico. En todo caso de ello deriva el modelo de administración estratégica presentado por clásicos como Steiner (1983), Mintzber (1990) y Lampel (2001), Glueck (1997), por nombrar algunos clásicos que han dejado grandes aportes en este particular. 
Desde la perspectiva de los autores consultados, y con la finalidad de sustentar teóricamente la investigación realizada, se asumen en el orden establecido las siguientes etapas: de la administración estratégica (Figura $\mathrm{N}^{\circ} 1$ )

\section{Figura $\mathrm{N}^{\circ} 1$ \\ Elementos de la Gerencia Estratégica}

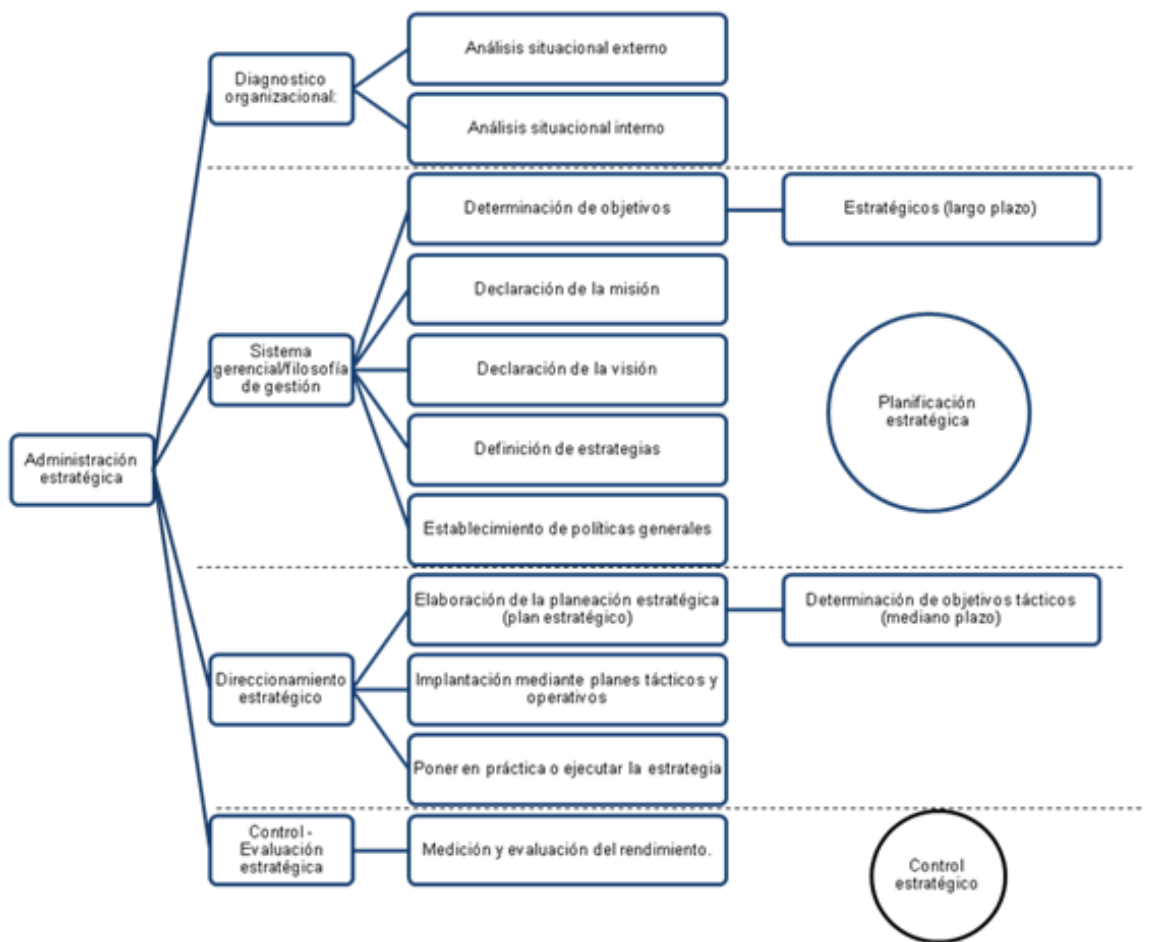

Fuente: Elaboración, a partir de Saavedra (2005), Chiavenato y Sapiro, (2011), David (2003), Thompson y Strckland, (2004).

\section{Diagnóstico organizacional: Análisis de los factores esenciales}

Kotler y Armstrong (2001) plantean que el diagnóstico organizacional parte de un análisis completo de la situación de la empresa, éste incluye la desagregación de los elementos externos a la organización, así como las consideración de aspectos internos; esto exige la realización de un sondeo del ambiente externo e interno a la 
Jorge González; Flor Salazar; Raúl Ortiz y Darwin Verdugo

Telos Vol. 21, No. 1 (2019). 242-267

organización, identificación de factores, variables, actores o elementos existentes que de manera directa e indirecta afectan las operaciones de la organización.

La importancia en la realización de este tipo de análisis, consiste en poder determinar de forma objetiva, en que aspectos la empresa tiene ventajas respecto a de su competencia y en qué aspectos necesita mejorar para ser competitiva.

En este orden de ideas David (2003), plantea la necesidad de una auditoría integral de la organización, para abarcar la evaluación del entorno (factores externos) y la evaluación de las fortalezas y debilidades de la empresa (factores internos).

\section{Factores del contexto externo a las organizaciones}

Las organizaciones están ubicadas en un complejo contexto económico, político, tecnológico, social, cultural y demográfico, a los cuales debe adaptar sus estrategias para alcanzar el cumplimiento de los objetivos organizacionales. Los factores del macroentorno se escapan del control de las organizaciones de modo positivo, negativo, directo o indirecto. La idea, de acuerdo con Hitt y col. (2004), es comprender factores externos que regulan los comportamientos de la organización, entre ellos, algunos de índole económica, política, tecnológicos, sociales, legales, entre otros. El reconocimiento y análisis de los factores externos es fundamental para garantizar una visión integral de la organización ante contextos complejos. Hitt y col. (2004)

Por su parte, David (2003), define las fuerzas externas como, situaciones que se traducen en cambios en la demanda de los consumidores, lo que puede afectar los tipos de productos y servicios desarrollados por la organización, el posicionamiento estratégico empresarial, las estrategias de segmentación del mercado y la selección de empresas para adquirir o vender.

A continuación se precisan cada uno de estos factores:

- Factores económicos 
Las dimensiones económicas del entorno son de vital importancia para las empresas y organizaciones. Las condiciones económicas representan una fuerza que repercute en las organizaciones, en su ciclo de vida, en las tasas de inflación e interés, periodo de crecimiento económico, recesión en el cual las personas disminuyen el consumo de bienes y servicios, depresión y recuperación, momento en que la economía pasa de la recesión a la prosperidad, entre otros D’Alessio (2008).

\section{- Factores políticos y legales}

Por otro lado, se encuentran factores políticos y legales, entendiendo que todas las organizaciones se encuentran inmersas en un ambiente político, el cual, según Hitt y col. (2004), es un terreno, donde las empresas compiten por influir en el rumbo de las políticas gubernamentales, mientras que en el ámbito legal son los gobiernos quienes establecen las leyes que rigen las actividades económicas de cualquier país.

Toda clase de organización puede verse afectada por las leyes y reglamentos que se impongan en una nación por la figura del gobierno; las empresas deben elaborar sus objetivos y metas organizaciones en base a una serie de leyes que tratan de regular y controlar el comportamiento de las mismas, por esta razón es importante tener conocimiento de las leyes impartidas para evitar la presencia de problemas que perjudiquen a la organización.

\section{- Factores tecnológicos}

Los factores tecnológicos, representan un elemento importante investigación, éstos tienen el poder de influir determinantemente en el contexto de cualquier sector económico, a través del desarrollo de nuevos productos que pueden salir al mercado a un menor costo y con la posibilidad de abrir las fronteras del sector ante los consumidores (Thompson y Strickland, 2004).

El desarrollo de tecnología aplicada a los productos de cualquier industria, origina un efecto directo sobre el nicho de mercado de la misma. Productos avanzados logran ampliar la base de clientes de una empresa, revitaliza el crecimiento y amplia el grado de diferenciación de sus productos.

\section{- Factores sociales}


Jorge González; Flor Salazar; Raúl Ortiz y Darwin Verdugo

Telos Vol. 21, No. 1 (2019). 242-267

Los factores sociales impactan de manera significativa la mayoría de los productos o servicios desarrollados, así como a los mercados y clientes, van desde los cambios sociales, demográficos, culturales y ambientales, desprendiéndose de estos cambios oportunidades y amenazas las cuales deben tomar en cuenta las grandes o pequeñas organizaciones, sean estas lucrativas o no (David, 2003).

Por su parte, Thompson y Strickland (2004), establecen que estos factores sociales pueden constituirse en fuerte promotores de cambios en la demanda de la industria. Para Hitt y col. (2004) los factores socio-culturales están referidos a los valores culturales de una sociedad, debido a que éstos representan las bases de toda organización social, y generalmente impulsan los cambios demográficos, económicos, tecnológicos y políticos.

En síntesis, el éxito de la gerencia estratégica para cualquier organización, depende en gran medida del análisis y diagnóstico que se ralice al contexto al cual pertenece la organización. En este sentido David (2003), plantea que la recolección y evaluación de la información sobre los competidores del sector es un factor básico para la formulación de estrategias con éxito.

\section{Factores internos a las organizaciones: elementos clave}

La necesidad en las organizaciones de realizar un diagnóstico interno, partiendo de la premisa de que toda empresa posee fortalezas y debilidades en sus áreas funcionales, es esencial, pues en toda organización existen elementos fuertes o débiles que deben identificarse (David, 2003).

Por ello, es fundamental la identificación de elementos que a lo interno de la organización y bajo un total control de ella, deben reconocerse y trabajar por su gestión adecuada. Este análisis permitirá determinar fortalezas y debilidades de la empresa respecto a sus competidores y, a partir de ellas, evaluar su capacidad para aprovechar las oportunidades y contrarrestar las amenazas. El conocimiento y dominio de las 
fortalezas de la organización demostrarán su superioridad frente a los competidores ya que se establecerán como ventajas competitivas.

\section{- Estructura organizativa}

Para conceptualizar la estructura organizativa, David (2003) la concibe como el patrón de diseño o modelo con el cual se organiza una entidad a fin de cumplir las metas propuestas y lograr el objetivo deseado. La estructura adecuada, permite comprender la diferencia entre una empresa y otra, siendo necesario, adoptar la estructura organizacional más conveniente a las prioridades o necesidades competitivas de la organización (es decir, la estructura deberá acoplarse y responder a la planeación), además debe reflejar la situación de la organización, por ejemplo, edad, tamaño, tipo de sistema de producción el grado de complejidad y dinamismo con el entorno.

\section{- Tecnología}

La tecnología es una variable interna de gran importancia en las organizaciones, puede ser asumida como variable ambiental, en el sentido que se asume como un componente del ambiente, que es adquirida por las organizaciones para incorporarlas en su ambiente de tarea, mientras que como variable organizacional forma parte del sistema interno de la organización. Dada su complejidad, se ha estudiado desde diversas perspectivas, llegando a la conclusión que como variable interna afecta La tecnología afecta el diseño organizacional, en función de ella se define la estructura organizativa de la empresa y las técnicas de producción.

Las empresas con operaciones estables necesitan estructuras diferentes a las requeridas por organizaciones con tecnología cambiante. La tecnología que adopta la empresa determinará su estructura y comportamiento organizacional.

\section{- Ventajas competitivas}

Para Porter (2006), la compañía puede obtener una ventaja competitiva a través de la gestión integrada de todos los elementos logísticos, incluyendo la 
Jorge González; Flor Salazar; Raúl Ortiz y Darwin Verdugo

Telos Vol. 21, No. 1 (2019). 242-267

infraestructura física, la tecnología, los procesos de negocio, las personas y el diseño de la organización. Una gestión óptima hace uso de los procesos de negocio, de su experiencia en el sector, de sistemas de información de primer nivel y de métodos de gestión eficaces, asegurando así su ventaja competitiva.

Una compañía puede ganar una ventaja competitiva reduciendo el tiempo destinado al ciclo de entrega de pedidos, que a la vez puede llevar a nuevas oportunidades comerciales. Otra ventaja competitiva consiste en reducir los inventarios, lo que resulta finalmente en una mejora de los flujos monetarios.

\section{- Sistemas de información}

Según David (2003), otro elemento a considerar en el diagnóstico interno organizacional, son los sistemas de información de la gerencia, los cuales generan la información que constituye la piedra angular de todas las organizaciones, pues proporcionan a todas las funciones del negocio la base para la toma de decisiones gerenciales. Por esta razón la información puede ser la causante de fortalezas y debilidades internas en una empresa.

El sistema de información es la herramienta de que dispone una organización para recaudar, almacenar, sintetizar y presentar la información veraz y pertinente para la toma de decisiones David (2003). Según Laudon y Laudon (2004), un sistema de información puede definirse técnicamente como un conjunto de componentes interrelacionados que recolectan o recuperan, procesan, almacenan y distribuyen información para apoyar la toma de decisiones y el control de una organización.

\section{- Talento humano}


En una organización es importante que la selección del personal tome como referencia las competencias académicas y la experiencia de los aspirantes puesto que esto redunda en mayor y mejor nivel de competitividad, productividad y rendimiento.

Cuando se alude al talento humano es importante destacar las relaciones interpersonales, las habilidades que tenga el personal de socializar, relacionarse y manejar conflictos. En organizaciones de base tecnológica, como las estudiadas, es necesario disponer de personal con habilidades y competencias que se orienten a la generación de conocimiento. Este talento debe ser su principal activo, y tener claramente definido el perfil general donde se incluyan conocimientos básicos, así como habilidades, destrezas, competencia que potencien el desarrollo y crecimiento de la organización.

\section{- Productos y servicios}

En organizaciones donde su naturaleza está constituida por la manufactura de insumos, con la finalidad de generar bienes, las funciones de producción ocupan un lugar fundamental en el éxito de sus actividades. Según David (2003), las funciones de producción y operaciones abarcan las entradas de insumos, su transformación y las salidas de productos que varían a través de las industrias y mercados.

\section{Elementos de la planificación estratégica: Sistema gerencial/Filosofía de gestión}

De acuerdo con David (2003), la planeación no es más que el proceso por medio del cual se determina si se debe realizar una actividad o tarea, se constituye en un mecanismo eficaz para alcanzar los objetivos propuestos, a la vez que ayuda a la empresa a obtener el efecto máximo de un esfuerzo dado, de igual forma permite reunir los recursos necesarios y realizar las tareas de la manera más eficiente poshible, con miras a evitar el menor desperdicio y obtener una utilidad justa.

La planeación ejerce un impacto positivo en el rendimiento de la empresa y del recurso humano, pues permite identificar y aprovechar las oportunidades externas, y al mismo tiempo minimiza el efecto de la amenaza. La planeación ofrece a una 
Jorge González; Flor Salazar; Raúl Ortiz y Darwin Verdugo

Telos Vol. 21, No. 1 (2019). 242-267

empresa la posibilidad de adaptarse a mercados cambiantes y poder trazar su rumbo desarrollándose como una organización proactiva más que reactiva.

En esta visión estratégica asumida para el análisis de las organizaciones, confluyen una serie de elementos o etapas que permiten precisar elementos representativos del entorno interno a la organización. Estas son las siguientes:

\section{$\checkmark$ Misión}

La misión de la organización se basa en una declaración duradera de sobre el propósito que distingue a una empresa de otra similar, en ella se identifica el alcance de la organización y debe describir los valores y prioridades de la misma establece la razón de ser de la organización y responde a la interrogante ¿cuál es nuestro negocio? "Quiénes somos, Qué hacemos y Por qué estamos aquí" (Chiavenato, 2011; Thompson y col. 2004; David, 2003).

De acuerdo con Para Thompson y col (2004) la misión ofrece información de la organización de carácter descriptivo, identifica los productos o servicios, especifica las necesidades que pretende satisfacer, los clientes o merados a los que se dedica atender y su propuesta para complacer al cliente.

La misión determina la razón de ser de una organización, es decir justifica su existencia, por lo que debe ser el norte que dirige la toma de decisiones y todas las actividades que se desarrollen deben estar encaminadas a este fin.

Los autores plantean que la misión debe contener los siguientes atributos: 1) Dar sentido al trabajo, 2) Concentrar los esfuerzos de la gente, 3) Concreta y clara, 4) Comprometedora, 5) Tangible, 6) Convincente, 7) Llegar al corazón de los empleados y también del cliente externo cuando este lo conoce, 8) Reflejar las creencias fundamentales de la organización.

\section{$\checkmark$ Visión}

Para definir la visión, se deben tener en cuenta algunos elementos fundamentales como son: 1) Panorama Futuro, el cual debe considerar el entorno 
económico y regulatorio en el que la organización va a competir, aspecto este que resulta determinante en los actuales momentos donde la apertura masiva de nuevos mercados obliga a revaluarse continuamente para ser competitivo. En este contexto económico es importante tener en cuenta aspectos como: el crecimiento del producto interno bruto, la inflación, las barreras arancelarias, la productividad, la balanza comercial, los recursos energéticos, la política industrial y los tratados internacionales y, 2) Marco competitivo, referido al conjunto de empresas que ofrecen productos o servicios competitivos entre sí. En este aspectos son frecuentes las alianzas estratégicas y privatizaciones, al igual que la compra de empresas con el fin de buscar dominio en el mercado. (Riveros, 2007)

La visión se formula desde la alta gerencia y debe poseer características como: coherente convincente; amplia y detallada; positiva e inspiradora y compartida por el equipo directivo, los colaboradores y los empleados.

\section{$\checkmark$ Objetivos}

Para el autor David (2003), los objetivos son declaraciones las cuales describen la naturaleza, el alcance, el estilo, así como los ideales de una organización para el mediano y largo plazo. En conjunto configuran una definición operativa de la visión, cuyo logro permitirá saber si se ha alcanzado o no. A su vez, cada objetivo estratégico debe responder (en la perspectiva en la que está planteada) haciendo las siguientes preguntas: ¿Qué se quiere lograr?, ¿Cuándo se debe lograr?, ¿Cómo se sabrá si se ha logrado?, éstos permiten determinar los logros que la organización quiere alcanzar en un plazo determinado para ser consistente con la orientación y propósitos estratégicos definidos en la misión. Los objetivos estratégicos deben ser: mensurables, comprensibles, congruentes, realistas, jerárquicos, estimulantes, coherentes, alcanzables, motivadores, planteados para el mediano o largo plazo.

Por su parte, Francés (2006), define los objetivos sobre la base de una acción, indicada por un verbo en infinitivo, y una variable estratégica, generalmente multidimensional; por ejemplo: incrementar la rentabilidad, reducir los costos, 
Jorge González; Flor Salazar; Raúl Ortiz y Darwin Verdugo

Telos Vol. 21, No. 1 (2019). 242-267

maximizar el valor. También pueden definir el estado que se desea alcanzar en relación con la variable (ser líderes en el mercado nacional, contar con estándares internacionales de desempeño).

\section{$\checkmark$ Políticas}

David (2003), define las políticas de una empresa como un proceso en el cual se utilizan las vías para hacer operativa las estrategias trazadas por una empresa, la política empresarial suele afectar a más de un área funcional, contribuyendo a cohesionar verticalmente la organización para el cumplimiento de los objetivos estratégicos, al igual que la estrategia. La política empresarial proporciona la orientación precisa para que los ejecutivos o mandos intermedios elaboren planes concretos de acción que permitan alcanzar los objetivos, en otras palabras, tiene como objetivo facilitar la creación y el desarrollo de empresas o industrias, es un proceso en el cual las personas:

Las políticas de una organización deben incluir las directrices, reglas y procedimientos con el propósito de propiciar esfuerzos encaminados al logro de los objetivos establecidos (David, 2003). Las políticas por lo general se establecen según cada actividad, mercadeo, finanzas producción, operación, diseño entre otros.

\section{$\checkmark$ Valores}

Según David (2003), los valores organizacionales son cualidades de la cultura de las empresas, que son jerarquizados o asumidos de preferencia porque son percibidos (en mayor o menor grado de conciencia), como elementos indispensables para alcanzar logros colectivos. Sin duda son aprendizajes estratégicos, componentes de la base de principios de la empresa, para nada estáticos, pues pueden estar sujetos a modificaciones, siempre y cuando ofrezcan nuevas oportunidades para la comprensión del fenómeno con el que se encuentre involucrado. 
Por su parte, Francés (2006), plantea los valores organizacionales como el marco ético-social dentro del cual la empresa lleva a cabo sus acciones; además forman parte de la cultura organizacional, estableciendo los limites en los cuales debe enmarcarse la conducta de los individuos pertenecientes a ella, tanto en el plano organizacional como en el plano personal, son atributos de las personas, de los grupos (como son las empresas), que guían su conducta.

En tal sentido, es relevante enfatizar que los valores están relacionados con las cualidades o actitudes deseadas por la empresa hacia sus empleados (por ejemplo: empatía, sinergia, entre otras). Es un factor principal para la consecución de los objetivos, además no todos son iguales para todas las empresas, ni tampoco son estáticos, es decir, pueden cambiar con el paso del tiempo

\section{$\checkmark \quad$ Estrategias}

Las estrategias se refieren a diversas elecciones administrativas que existen entre diversas opciones, es decir se constituyen en el medio para lograr el fin (objetivo deseado), son acciones que se definen para consolidar el logro de los resultados propuestos (Chiavenato, 2011; Thompson y Strickland, 2004; David, 2003).

Para David (2003) las estrategias son medios por los cuales se logran los objetivos; incluyen expansión, diversificación, adquisición y desarrollo, penetración en el mercado y reducción de costos. Son acciones que requieren toma de decisiones por parte de la gerencia ya que estas tendrán efecto en las funciones de la empresa.

\section{Direccionamiento estratégico}

El direccionamiento estratégico se define como la formulación de las finalidades y propósitos de una organización, donde se consignan los objetivos definidos para un largo plazo que apuntan a la perdurabilidad, sostenibilidad y crecimiento de la misma que sirve de marco referencial para los objetivos y lineamientos consagrados en el plan estratégico.

El Direccionamiento estretegico nace con la necesidad que tiene la alta gerencia de orientar y enriquecer las competencias gerenciales y conducir a las 
organizaciones en la gestión integral y afianzar la competitividad del negocio con el conocimiento y aplicación de métodos eficaces.

Según Dess y Lumpkin (2003), el proceso de dirección estratégica considera el transcurso de tres etapas: la determinacion de los objetivos estratégicos (análisis), la formulación estratégica o implementacion de los planes tacticos y operativos (elección, decisión), y la implantación o ejecucion de la estregtegia (acción). Esto significa que luego de formular los Objetivos Estratégicos y de realizar el análisis situacional se proceden a diseñar estrategias de mejoramiento que nos van a permitir lograr los objetivos propuestos.

En cada una de las etapas del proceso de direccionamiento estratégico se debe establecer una adecuada integración de la organización con el entorno, tomando como base de su cambio y mejora continua, las exigencias del sistema o entorno.

Por esta razón, las empresas deben ejecutar sus decisiones de forma contundente, para responder a los cambios del entorno, entre las cuales se pueden destacar: la practicidad de los procesos, y el desarrollo de una cultura de conocimiento, que permita la innovación constante con enfoque en la productividad de alto nivel.

\section{Proceso de evaluación estratégica}

La evaluación de la estrategia según David (2003), se vuelve cada vez más difícil con el tiempo por muchas razones. Las economías domésticas y mundiales eran más estables en años anteriores, los ciclos de vida de los productos eran más largos, los ciclos de desarrollo de productos eran más prolongados, el avance tecnológico era más lento, los cambios ocurrían con menor frecuencia, había menos competidores, las empresas eran débiles y había más industrias reguladas.

\section{Medición del desempeño}

La medición de desempeño, en términos generales, es un esfuerzo sistemático aplicado en una organización para evaluar su gestión orientada al cumplimiento de su misión, a partir de la optimización de sus procesos. Dicha 
evaluación resulta útil para, validar y redefinir las actividades de la empresa (tal como la selección y capacitación), asi como para brindar información a los empleados que deseen mejorar su futuro rendimiento.

El desempeño de una organización depende en mucho del desempeño de su personal. Para que pueda determinarse la contribución de cada individuo a la organización, es necesario tener en cuenta un programa formal de evaluación con los objetivos claramente enunciados y un sistema bien organizado para alcanzarlos

\section{Evaluación del rendimiento}

Otra actividad importante de evaluación de la estrategia es la medición del rendimiento de la empresa. Esta actividad incluye la comparación de los resultados esperados con los resultados reales, la investigación de las desviaciones de los planes, la evaluación del desempeño individual y el examen del progreso hacia el cumplimiento de los objetivos establecidos. Los objetivos tanto a largo como a corto plazo se utilizan con frecuencia en este proceso. Los criterios para la evaluación de las estrategias deben ser cuantificables y verificables con facilidad

\section{Toma decisiones/Acciones correctivas}

De acuerdo con diversas investigaciones y enfoques teóricos, la toma de decisiones puede definirse como: "Un proceso amplio que puede incluir tanto la evaluación de las alternativas, el juicio, como la elección de una de ellas (...)” (Artieta y González, 1998:368). En otras palabras, la toma de decisiones hace referencia a la capacidad cognitiva para elegir; lo que involucra: análisis, categorización, juicios probabilísticos, construcción de alternativas y decisión.

Para David (2003), la toma de decisiones es un proceso intencional que combina el análisis de la información, la confrontación de alternativas, la valoración de las opciones y, finalmente, la toma de la decisión. Se trata de formarse en el uso de métodos sistemáticos, es decir, estructurados y coherentes con el campo disciplinar, técnico o académico de la decisión; elegir entre las posibilidades, previamente 
Jorge González; Flor Salazar; Raúl Ortiz y Darwin Verdugo

Telos Vol. 21, No. 1 (2019). 242-267

identificadas, la mejor forma de proceder valorando las implicaciones y consecuencias de tal forma que se revele el nivel de compromiso con ella. Un método sistemático se funda en datos, en recabar información de calidad, verificarla y contrastarla con otras del campo de producción específico y de otros. Se trata de redescubrir, de acuerdo con la experiencia y la práctica opciones y caminos más consistentes con el tipo de decisión a tomar.

\section{Reflexiones finales}

La gerencia estratégica es una herramienta fundamental en cualquier tipo de organizaciones, en esta oportunidad se estudiaron elementos esenciales de esta herramienta desde la perspectiva teórica considerando visiones clásicas de autores especializados en el área.

El análisis estratégico genera una perspectiva sobre el presente y futuro de la organización, dejando en claro las oportunidades que ofrece el entorno y las competencias de la empresa. Es importante generar y seguir procesos lógicos de desarrollo estratégico. Sin embargo, la experticia e intuición de los gerentes es fundamental, puesto que seguir un proceso metodológico no garantiza el éxito organizacional.

Todas las organizaciones poseen una estrategia, que las dirige hacia algún rumbo, por ello requieren de una estrategia formal, derivada de un análisis del ambiente, de valorar las fortalezas y las debilidades e identificar las oportunidades en donde la organización tuviera una ventaja competitiva.

Por tanto, es evidente la necesidad de que las empresas adopten métodos o técnicas acordes a los nuevos tiempos que les permitan sobrevivir e incluso crecer y consolidarse en estos ambientes que son cada vez más turbulentos y complejos. Es aquí donde, sin duda, gerencia estratégica tendrá un papel más preponderante.

La gerencia estratégica es de vital importancia en todas las organizaciones. El proceso de gerencia estratégica se aplica de igual forma tanto a empresas grandes como pequeñas. Desde el momento de su concepción, toda organización posee una estrategia. 
Tambien es importante destacar que los objetivos, las metas, las estrategias y las políticas no tienen muchas posibilidades de éxito, si los gerentes no se encuentran motivados para ejecutar las estrategias tan pronto ellas se formulan y ocurran cambios que los gerentes lo reconozcan como necesario y beneficioso yque varien en el transcurso del tiempo.

Es de destacar que la principal, ventaja competitiva es el talento humano creativo y altamente especializado y, sin duda la tecnología como medio y fin de conducir desarrollos significativos con grandes aportaciones no solo para las empresas, sino para el país y la región en la cual se desenvuelven.

Finalmente, la gerencia estratégica permite la toma de decisiones del gerente, las cuales deben asumirse como transformaciones, que conduzcan a las organizaciones por nuevos rumbos, nuevos procesos, nuevos objetivos, nuevas estrategias, nuevas tecnologías e incluso nuevos riesgos; y a nuevos comportamientos y actitudes del capital humano. Todo ello, utilizando herramientas que permitan un desarrollo gerencial dirigido e incrementar la habilidad del liderazgo y resaltar los valores institucionales.

\section{Referencias Bibliográficas}

Artieta, Isabel y González, María. (1998). La toma de decisiones. Introducción a la Psicología del pensamiento, Editorial Trotta. España.

Betancourt, José R. (2006). Navegando hacia el cuarto paradigma. Clima y compromiso organizacional. Editorial Granica. México.

Chiavenato, Idalberto y Sapiro, Arâo (2011). Planeación estratégica. Fundamentos y aplicaciones. Segunda Edición. McGraw-Hill Education. México.

Chiavenato, Idalberto. (2011). Introducción a la teoría general de la administración. Editorial McGraw Hill. México.

Chiavenato, Idalberto (2001). Administración. Proceso Administrativo. Teoríaproceso-practica. Tercera edición. McGraw Hill. Colombia

David, Fred (2003). Conceptos de Administración estratégica. Novena edición. Editorial Pearson Hall. México.

D'alessio, Fernando. (2008). El Proceso Estratégico: Un Enfoque de Gerencia. Pearson. Prentice Hall. México.

Dess, Gregory y Lumpkin, Tom. (2003). Dirección estratégica: creando ventajas competitivas. McGraw Hill. España.

Francés, Antonio (2006). Estrategia para la empresa en América latina. Segunda edición .Ediciones IESA. Venezuela. 
Jorge González; Flor Salazar; Raúl Ortiz y Darwin Verdugo

Telos Vol. 21, No. 1 (2019). 242-267

García Jambel. (2010). Pensamiento Estratégico: herramienta de competitividad para una orientación Gerencial del nuevo milenio. Extraído de: http://www.ucla.edu.ve/dac/revistateacs/articulos/Rev4-Ens3-Garcia.pdf consulta: 05/11/2018.

Hitt, Michael; Duane Ireland y Hoskisson, Robert. (2004). Administración estratégica. Competitividad y conceptos de globalización. Quinta edición. Editorial Thomson. México.

Kotler Philip; y Armstrong Gary. (2001) Fundamentos de marketing. Pearson Education. Mexico.

Lampel, Joseph. (2001) Strategy Safari: A Guided Tour Through The Wilds of Strategic Mangament. The free press. USA.

Laudon, Kenneth y Laudon, Jane. (2004). Sistema de Información Gerencial. Pearson Education. Mexico.

Mintzberg, Henry. (1990). Strategy formation: Schools of thought. En: Fredrickson, James (Ed.). Perspectives on strategic management. Harper Business. USA.

Porter, Michael E. (2006). Estrategia y Ventaja Competitiva. Líderes del Management. Primera edición. Ediciones Deusto. España.

Rivas, Milaydis y Velázquez, Reynaldo. (2009). La gestión integrada, estratégica, por competencias y procesos. Enfoques contemporáneos en la gestión de los recursos humanos. Extraído de: https://econpapers.repec.org/article/ervcontri/y 3a2009_3ai_3a200907 3a13.htm Consulta 12/08/2014.

Riveros, Pablo. (2007). Sistema de gestión de la calidad del servicio. Sea líder en mercados altamente competitivos. 3ra Edición. ECOE Ediciones. Colombia. Saavedra, Juan. (2005). Administración estratégica: evolución y tendencias. Revista Economía y Administración. Extraído de: http://www2.udec.cl/ rea/REVISTA\%20PDF/Rev64/art3rea64.pdf consulta: $12 / 08 / 2018$.

Senge, Peter. (2009). La Quinta Disciplina. El arte y la práctica de la organización abierta al aprendizaje. Editorial Profit. España.

Steiner, George. (1983). Planeación estratégica: lo que todo director debe saber . Compañía editorial continental S.A. México.

Thompson, Arthur y Strickland, A.J (2004). Administración Estratégica. Undécima edición. McGraw-Hill. México. 